\title{
Enantiospecific synthesis of [2.2]paracyclophane-4-thiol and derivatives
}

\author{
Gareth J. Rowlands ${ }^{*}, 1$ and Richard J. Seacome ${ }^{2}$
}

\section{Full Research Paper}

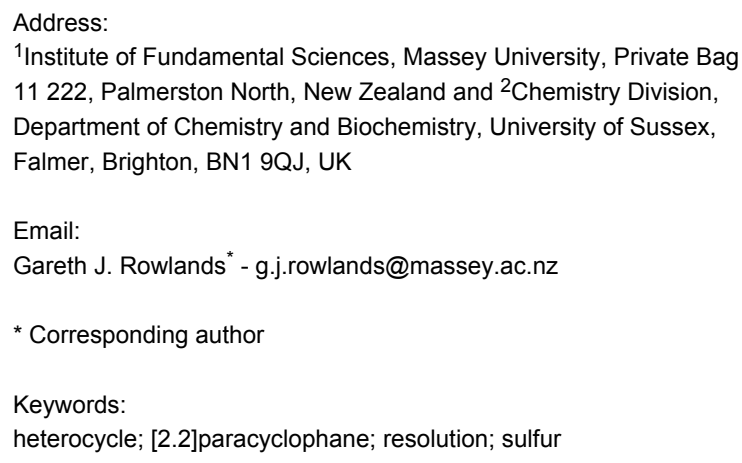

${ }^{1}$ Institute of Fundamental Sciences, Massey University, Private Bag 11 222, Palmerston North, New Zealand and ${ }^{2}$ Chemistry Division, Department of Chemistry and Biochemistry, University of Sussex, Falmer, Brighton, BN1 9QJ, UK

\section{Email:}

Gareth J. Rowlands* - g.j.rowlands@massey.ac.nz

* Corresponding author

Keywords:

heterocycle; [2.2]paracyclophane; resolution; sulfur

\author{
Beilstein Journal of Organic Chemistry 2009, 5, No. 9. \\ doi:10.3762/bjoc.5.9 \\ Received: 15 January 2009 \\ Accepted: 28 February 2009 \\ Published: 12 March 2009 \\ Editor-in-Chief: J. Clayden \\ (C) 2009 Rowlands and Seacome; licensee Beilstein-Institut. \\ License and terms: see end of document.
}

\section{Abstract}

This paper describes a simple route to enantiomerically enriched [2.2]paracyclophane-4-thiol via the stereospecific introduction of a chiral sulfoxide to the [2.2]paracyclophane skeleton. The first synthesis of an enantiomerically enriched planar chiral benzothiazole is also reported.

\section{Introduction}

[2.2]Paracyclophane $(\mathbf{1} ; \mathrm{R}=\mathrm{H})$ is a fascinating compound comprising of two eclipsing benzene rings that are held in place by two ethyl bridges at the para positions (Figure 1). The close proximity of the arene moieties results in strong electronic and structural interactions between the two rings and between substituents appended to each layer $[1,2]$. The resulting unique properties have led to derivatives of [2.2]paracyclophane being employed in a wide range of disciplines including polymer, material and electronic chemistry [3-9]. Whilst enantiomerically pure derivatives have been utilised in chiral catalysis [10, 11] and as probes for biological recognition processes [12-14], the full potential of these systems has not been realised due to the difficulties encountered when trying to access enantiomerically pure [2.2]paracyclophane derivatives [15].

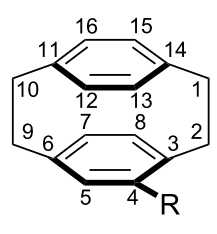

1

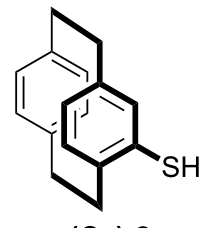

$\left(S_{p}\right)-2$
Figure 1: [2.2]Paracyclophane (1) showing standard numbering and [2.2]paracyclophane-4-thiol (2).

Chiral sulfur [2.2]paracyclophane derivatives are beginning to attract attention due to the great potential such compounds exhibit [16-18]. Non-cyclophane-based thiophenol derivatives 
have been employed in the nucleophilic addition of thioacetals to suitable electrophiles $[19,20]$, sigmatropic rearrangements [21] and as either thiyl radical precursors [22] or as a source of hydrogen in radical chemistry [23]. With the appropriate sulfur derivative, stereoselective variants of all these transformations can be envisaged.

Currently, there are few examples of sulfur containing [2.2]paracyclophane compounds; aryl sulfonylation and the related sulfenylation facilitates the synthesis of sulfonic acids, sulfonamides and protected thiols [24-26] whilst directed metallation has allowed the formation of various sulfides [27-30]. Very few methodologies allow the synthesis of simple chiral monosubstituted thiols such as [2.2]paracyclophane-4thiol 2 (Figure 1); the first reported preparations of racemic 2 were the conversion of 4-hydroxy[2.2]paracyclophane to the desired compound via a Newman-Kwart reaction or the direct reaction of 4-lithio[2.2]paracyclophane with sulfur [17]. Use of enantiomerically pure 4-hydroxy[2.2]paracyclophane or application of our own sulfoxide-metal exchange protocol [31] would permit enantiospecific variants of either route, but neither has been reported. An elegant entry to a variety of racemic alkyl sulfides and sulfoxides by an $S_{E} A r$ reaction mediated by a sulfonium salt has recently been divulged [16]. The only reported synthesis of enantiomerically pure [2.2]paracyclophane-4-thiol entails the palladium-mediated addition of triisopropylsilanethiol to a triflate formed from previously resolved $(R)-4$ hydroxy[2.2]paracyclophane [18]. We are developing a 'toolbox' for the synthesis of enantiomerically enriched [2.2]para- cyclophane derivatives based on the chemistry of [2.2]paracyclophane sulfoxides [15,31-33]. This methodology has allowed us to develop routes to enantiomerically pure 4-monosubstituted [2.2]paracyclophanes [31] along with a range of disubstituted derivatives [33]. The basis of the strategy is the stereospecific introduction of a sulfoxide to [2.2]paracyclophane to give readily separable diastereoisomers, thus resolving the planar chirality [34]. The sulfoxide moiety is used to direct further elaboration of the [2.2] paracyclophane framework or is displaced via sulfoxide-metal exchange. We were interested in modifying this methodology to permit the synthesis of enantiomerically enriched [2.2]paracyclophane-4-thiol and related compounds.

\section{Results and Discussion}

The synthesis of $\left(S_{\mathrm{p}}\right)-\mathbf{2}$ is depicted in Scheme 1. Key to the success of this strategy was the resolution of the planar chirality of [2.2]paracyclophane by incorporation of the tert-butylsulfinyl moiety to give the diastereoisomers $\left(S_{\mathrm{p}}, R_{\mathrm{S}}\right)-\mathbf{5}$ and $\left(R_{\mathrm{p}}, R_{\mathrm{S}}\right)-\mathbf{5}$. Standard iron-catalysed bromination of 1 gave $( \pm)-4-$ bromo[2.2]paracyclophane 3 in good yield $[35,36]$. Halogenlithium exchange and addition to Ellman's $(R)$-tert-butyl tertbutanethiosulfinate [37] 4 furnished a $1: 1.4$ mixture of $\left(S_{\mathrm{p}}, R_{\mathrm{S}}\right)$ 5 and $\left(R_{\mathrm{p}}, R_{\mathrm{S}}\right)-\mathbf{5}$ in a combined $72 \%$ yield [33]. The two diastereoisomers are readily separable by standard column chromatography, with the diastereoisomer $\left(S_{\mathrm{p}}, R_{\mathrm{S}}\right)-\mathbf{5}$ being eluted first. As $(R)-4$ was prepared with an ee of $80 \%$, as judged by optical rotation, we assume that each diastereoisomer displays an $e e$ of $80 \%$. As the sulfinylation reaction proceeds with inversion at

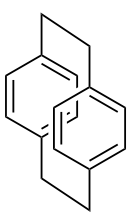

1

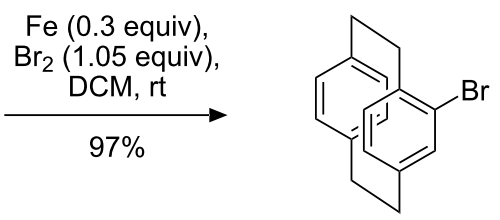

(士)-3

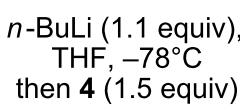

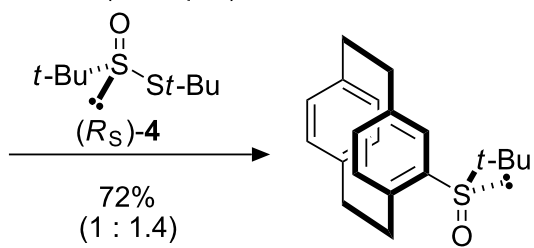

$\left(S_{\mathrm{p}}, R_{\mathrm{S}}\right)-5$

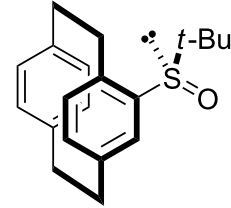

$\left(R_{\mathrm{p}}, R_{\mathrm{S}}\right)-5$

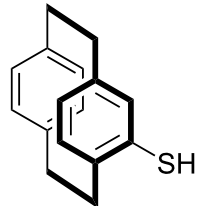

$\left(S_{\mathrm{p}}\right)-2$

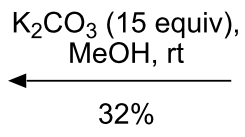

$32 \%$

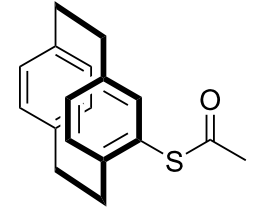

$\left(S_{p}\right)-7$

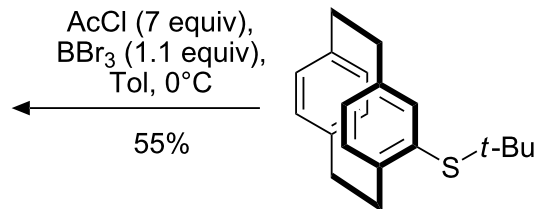

$\left(S_{p}\right)-6$

Scheme 1: Conversion of [2.2]paracyclophane to enantiomerically enriched [2.2]paracyclophane-4-thiol. 
sulfur, the two diastereoisomers only differ by the chirality of the [2.2]paracyclophane therefore allowing the facile resolution of the planar chirality. The assignment of configuration is based on a combination of X-ray studies $[33,38]$, formation of all stereoisomers and analogy to our previous tolylsulfinyl chemistry $[31,39]$.

Unlike the previously prepared 4-tolylsulfinyl[2.2]paracyclophane [31], direct sulfoxide-metal exchange was not possible with the tert-butyl derivative [38]; presumably, the tert-butyl group and the lower ring of the [2.2]paracyclophane moiety shield the sulfur from attack. As a result a stepwise procedure for the conversion of $\left(S_{\mathrm{p}}, R_{\mathrm{S}}\right)-5$ to [2.2]paracyclophane-4-thiol $\left(S_{\mathrm{p}}\right)$-2 was investigated (Scheme 1). The first step, the reduction of $\left(S_{\mathrm{p}}, R_{\mathrm{S}}\right)-5$ to sulfide $\left(S_{\mathrm{p}}\right)-\mathbf{6}$, proved the most problematic; use of a large excess of trichlorosilane and triethylamine resulted in deoxygenation in moderate yield after recrystallisation [40]. By comparison, reduction of the less hindered aryl sulfoxide, $\left(R_{\mathrm{p}}, S_{\mathrm{S}}\right)$-4-bromo-13-p-tolylsulfinyl[2.2]paracyclophane, occurs efficiently in $98 \%$ yield suggesting the tert-butyl group is the source of the problem [39]. Exchange of the tertbutyl group for an acetyl group was achieved by reaction of a mixture of $\left(S_{\mathrm{p}}\right)-\mathbf{6}$ and acetyl chloride in toluene with boron tribromide [41]. The resulting thioacetic acid $S$-[2.2]paracyclophane ester $\left(S_{\mathrm{p}}\right)-7$ readily undergoes simple base-catalysed hydrolysis to give the desired $(S)-(+)-[2.2]$ paracyclophane-4thiol $\left(S_{\mathrm{p}}\right)-2$.

There are two advantages to our methodology compared to the previously reported syntheses of [2.2]paracyclophane thiols; the first is that resolution of the planar chirality is complicit in the addition of the sulfur moiety and does not require resolution of any precursors. Secondly, the sulfinyl moiety permits further functionalisation of the [2.2]paracyclophane skeleton [33]. It is the latter reason that prompted the synthesis of the thiol via the tert-butyl derivative and not by direct sulfoxide-metal exchange; whilst this route would have delivered $\mathbf{2}$ more rapidly it would not have permitted elaboration of the [2.2]paracyclophane framework. The utility of the tert-butyl derivative is demonstrated in the synthesis of the planar chiral benzothiazole $\left(R_{\mathrm{p}}\right)-\mathbf{1 0}$ (Scheme 2).

Benzothiazoles are important heterocycles having found use as dyes, pharmaceuticals and ligands in catalysis [42]. Planar chiral heterocycles are still rare but show considerable potential as probes in stereocontrolled recognition processes in biological systems as highlighted by Gmeiner [12-14] and as ligands or catalysts [43-47]. We have previously prepared planar chiral benzimidazoles [32] and wanted to extend the range of heterocycles that could be accessed.

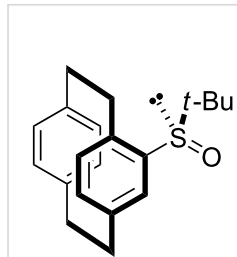

$\left(R_{\mathrm{p}}, R_{\mathrm{S}}\right)-5$

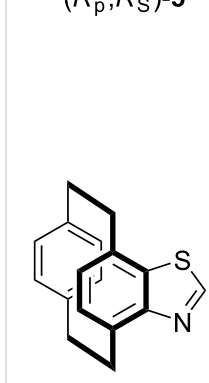

$\left(R_{\mathrm{p}}\right)-10$ i. $n$-BuLi (2.2 equiv), THF,

$\mathrm{NaBH}_{4}\left(9+4\right.$ equiv), $\mathrm{Bu}_{4} \mathrm{NI} \rightarrow t$-Bu ( 0.9 equiv), $\mathrm{rt}$

$66 \%$
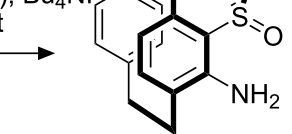

$\left(R_{\mathrm{p}}, R_{\mathrm{S}}\right)-8$
$-78{ }^{\circ} \mathrm{C}$ then $\mathrm{TsN}_{3}$ ( 2.5 equiv)

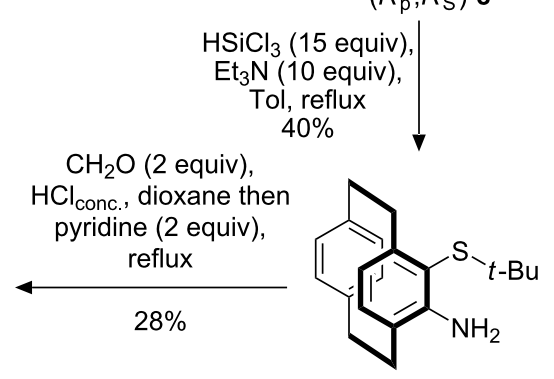

$\left(R_{\mathrm{p}}\right)-\mathbf{9}$
Scheme 2: Synthesis of [2.2](4,7)benzo[d]thiazoloparacyclophane $\left(R_{\mathrm{p}}\right)-10$.

Diastereoisomer $\left(R_{\mathrm{p}}, R_{\mathrm{S}}\right)$-5 was functionalised by sulfinyldirected ortho lithiation with $n$-butyllithium followed by reaction with tosyl azide. The resulting azo[2.2]paracyclophane was reduced in situ to give the amine $\left(R_{\mathrm{p}}, R_{\mathrm{S}}\right)-\mathbf{8}$ in good yield for the two steps (Scheme 2). Trichlorosilane-mediated deoxygenation proceeded uneventfully to furnished amino sulfide $\left(R_{\mathrm{p}}\right) \mathbf{- 9}$. Simultaneous sulfide deprotection and thiazole formation was achieved by treating $\left(R_{\mathrm{p}}\right)-9$ with concentrated hydrochloric acid, paraformaldehyde and pyridine [48]. Although the yield of $\left(R_{\mathrm{p}}\right)$-10 is not yet satisfactory, it shows the potential of our methodology for the formation of these valuable heterocycles.

In conclusion, we have developed a straightforward method for the synthesis of enantiomerically enriched [2.2]paracyclophane4-thiol that does not rely on the resolution of precursors to the introduction of the sulfur moiety. Furthermore, we have shown that this methodology has the potential to produce a wide-range of thiol derivatives and this has permitted the first synthesis of a planar chiral benzothiazole, $[2.2](4,7)$ benzo[d] thiazoloparacyclophane. The use of these thiols in asymmetric synthesis is currently being investigated and will be reported in due course.

\section{Experimental}

NMR spectra were recorded on a Bruker $400 \mathrm{MHz}$, Bruker 300 $\mathrm{MHz}$, Varian $500 \mathrm{MHz}$ or Varian $400 \mathrm{MHz}$ using residual isotopic solvent as internal reference. Infrared spectra were recorded on a Perkin-Elmer 1600 Fourier Transform spectrometer. Mass spectra and exact mass data were recorded by Dr. Ali Abdul-Sada at the University of Sussex or by the EPSRC national mass spectrometry service, Swansea. Melting points were recorded on a Gallenkamp melting point apparatus and are 
uncorrected. Optical rotation was recorded on a Perkin Elmer 241 polarimeter using a sodium lamp emitting at $589 \mathrm{~nm}$. All samples were measured in chloroform $(c=1)$ in a $10 \mathrm{~cm}$ cell and an average taken of 10 readings; average temperature was $27^{\circ} \mathrm{C}$. Glassware was oven dried and reactions were performed under an inert atmosphere of nitrogen or argon where applicable. Chromatography refers to flash column chromatography on Merck Kieselgel 60 (230-400 mesh) or Fischer Davisil 60 silica gel unless otherwise stated. TLC refers to analytical thinlayer chromatography performed using pre-coated glass-backed plates (Merck Kieselgel $60 \mathrm{~F}_{254}$ ) and visualised with ultraviolet light, iodine, acidic ammonium molybdate (IV), acidic ethanolic vanillin, aqueous potassium manganate(VII), ninhydrin or acidic anisaldehyde as appropriate. Petrol refers to redistilled petroleum ether $\left(60-80^{\circ} \mathrm{C}\right)$, and ether to diethyl ether. Ether and THF were distilled from sodium-benzophenone ketyl, toluene from $4 \AA$ molecular sieves or calcium chloride. Dioxane was stored over sodium wire and DMF was stored over $4 \AA$ molecular sieves.

\section{( \pm )-4-Bromo[2.2]paracyclophane (3)}

All stages of this reaction were performed in the dark by covering the flasks with aluminium foil. Bromine $(7.8 \mathrm{~mL}, 0.15$ mol, 1.05 equiv) was dissolved in DCM $(1.5 \mathrm{~L}) .10 \%$ of the solution $(150 \mathrm{~mL})$ was transferred to a flask containing iron filings ( $2.4 \mathrm{~g}, 0.04 \mathrm{~mol}, 0.3$ equiv) and stirred at rt for $1.5 \mathrm{~h}$. A solution of [2.2]paracyclophane ( $30.0 \mathrm{~g}, 0.14 \mathrm{~mol}, 1.0$ equiv) in DCM $(2.8 \mathrm{~L})$ was added and the suspension stirred for $20 \mathrm{~min}$. The remaining solution of bromine in DCM $(1350 \mathrm{~mL})$ was added via cannula over 30 minutes. After 5 min TLC indicated complete reaction and saturated aqueous $\mathrm{NH}_{4} \mathrm{Cl}$ solution $(5.0 \mathrm{~L})$ was added. The aqueous phase was extracted with DCM $(3 \times 1$ L) and the combined organic extracts washed with aqueous $\mathrm{Na}_{2} \mathrm{~S}_{2} \mathrm{O}_{3}$ solution $(10 \% \mathrm{w} / \mathrm{v} ; 1 \mathrm{~L})$ then dried $\left(\mathrm{MgSO}_{4}\right)$. The solvent was removed to give 3 as a white powder (40.2 g, 97\%); $\mathrm{mp}=134-135{ }^{\circ} \mathrm{C}$ (lit. [35]: 136-138 ${ }^{\circ} \mathrm{C}$ ); $v_{\max }$ (film) 3055, 2987, 1422, 1265, 896, 739 and $705 \mathrm{~cm}^{-1} ; \delta_{\mathrm{H}}(300 \mathrm{MHz}$, $\left.\mathrm{CDCl}_{3}\right) 7.15(1 \mathrm{H}, \mathrm{d}, J=9.0 \mathrm{~Hz}, \mathrm{H}-13), 6.56(1 \mathrm{H}, \mathrm{d}, J=9.0 \mathrm{~Hz}$, $\mathrm{H}-8), 6.51-6.46$ (4H, m, H-5, H-7, H-15 and H-16), $6.44(1 \mathrm{H}$, $\mathrm{d}, J=9.0 \mathrm{~Hz}, \mathrm{H}-12), 3.46(1 \mathrm{H}, \mathrm{ddd}, J=10.2,7.7,2.1 \mathrm{~Hz}, \mathrm{H}-2$ endo), 3.24-3.15 (1H, m, H-1 endo), 3.98-3.13 (5H, m, H-1 exo, $2 \times \mathrm{H}-9$ and $2 \times \mathrm{H}-10), 2.95-2.77(1 \mathrm{H}, \mathrm{m}, \mathrm{H}-2$ exo $) ; \delta_{\mathrm{C}}(75$ $\left.\mathrm{MHz}, \mathrm{CDCl}_{3}\right) 142.0,139.7,139.5,137.6,135.4,133.7,133.4$, $133.3,132.6,131.8,129.1,127.4,36.2,35.9,35.2,33.8$.

\section{$\left(R_{\mathrm{p}}, R_{\mathrm{S}}\right)-(-)-4-$ tert-Butylsulfinyl[2.2]paracyclo-} phane $\left[\left(R_{\mathrm{p}}, R_{\mathrm{S}}\right)-5\right]$ and $\left(S_{\mathrm{p}}, R_{\mathrm{S}}\right)-(-)$-4-tert-

\section{Butylsulfinyl[2.2]paracyclophane [( $\left.\left.S_{\mathrm{p}}, R_{\mathrm{S}}\right)-5\right]$}

To a solution of $( \pm)$-4-bromo[2.2]paracyclophane (5.50 g, 19.16 mmol, 1.0 equiv) in THF $(180 \mathrm{~mL})$ at $-78{ }^{\circ} \mathrm{C}$ was added $n$-BuLi (2.5 M in hexanes; $8.5 \mathrm{~mL}, 21.08 \mathrm{mmol}, 1.1$ equiv) dropwise over $15 \mathrm{~min}$. After $45 \mathrm{~min},(R)$-tert-butyl tert-butanethiosulfinate $(R)-4$ ( $80 \% e e ; 5.57 \mathrm{~g}, 28.74 \mathrm{mmol}, 1.5$ equiv) was added as a solid and the reaction stirred at $\mathrm{rt}$ overnight. The solvent was removed and the resulting residue purified by chromatography $\left(\mathrm{Et}_{2} \mathrm{O} / n\right.$-heptane gradient) to yield $\left(R_{\mathrm{p}}, R_{\mathrm{S}}\right)-\mathbf{5}(1.79$ $\mathrm{g}, 30 \%)$ and $\left(S_{\mathrm{p}}, R_{\mathrm{S}}\right)-5(2.51 \mathrm{~g}, 42 \%)$.

\section{$\left(R_{\mathrm{p}}, R_{\mathrm{S}}\right)-(-)-4$-tert-Butylsulfinyl[2.2]paracyclophane $\left[\left(R_{\mathrm{p}}, R_{\mathrm{S}}\right)-5\right]$}

mp $124-126^{\circ} \mathrm{C} ;[\alpha]_{\mathrm{D}}-39.6\left(c 1, \mathrm{CHCl}_{3}\right.$ ) (assumed $80 \%$ ee see text); $v_{\max }$ (film) 2962, 2926, 1585, 1456, 1473, 1500, 1170, $1054,1024,908$ and $847 \mathrm{~cm}^{-1} ; \delta_{\mathrm{H}}\left(500 \mathrm{MHz}, \mathrm{CDCl}_{3}\right) 7.02$ $(1 \mathrm{H}, \mathrm{s}, \mathrm{H}-5), 6.83(1 \mathrm{H}, \mathrm{d}, J=7.5 \mathrm{~Hz}, \mathrm{H}-13), 6.62(1 \mathrm{H}, \mathrm{d}, J=7.5$ $\mathrm{Hz}, \mathrm{H}-7), 6.54(1 \mathrm{H}, \mathrm{d}, J=8.0 \mathrm{~Hz}, \mathrm{H}-12), 6.52(2 \mathrm{H}, \mathrm{s}, \mathrm{H}-15$, $\mathrm{H}-16), 6.48(1 \mathrm{H}, \mathrm{d}, J=8.0 \mathrm{~Hz}, \mathrm{H}-8), 3.54(1 \mathrm{H}, \mathrm{ddd}, J=13.5$, $12.3,2.5 \mathrm{~Hz}, \mathrm{H}-2$ endo), 3.27 (1H, ddd, $J=13.0,9.1,5.5 \mathrm{~Hz}$, $\mathrm{H}-1$ endo), 3.16-3.06 (5H, m, H-1 exo, $2 \times \mathrm{H}-9$ \& $2 \times \mathrm{H}-10)$, $2.89(1 \mathrm{H}, \mathrm{ddd}, J=10.0,8.9,5.5 \mathrm{~Hz}, \mathrm{H}-2$ exo $), 1.05(9 \mathrm{H}, \mathrm{s}$, $t$-Bu); $\delta_{\mathrm{C}}\left(125 \mathrm{MHz}, \mathrm{CDCl}_{3}\right) 140.7$ (C), 139.5 (C), $139.0(\mathrm{C})$, $138.9(\mathrm{C}), 138.9(\mathrm{C}), 136.0(\mathrm{CH}), 134.6(\mathrm{CH}), 133.1(\mathrm{CH})$, $132.7(\mathrm{CH}), 132.6(\mathrm{CH}), 132.3(\mathrm{CH}), 130.3(\mathrm{CH}), 56.6(\mathrm{C})$, $35.2\left(\mathrm{CH}_{2}\right), 35.1\left(\mathrm{CH}_{2}\right), 34.7\left(\mathrm{CH}_{2}\right), 33.6\left(\mathrm{CH}_{2}\right), 22.7\left(\mathrm{CH}_{3}\right)$; $m / z(\mathrm{EI}+) 256[\mathrm{M}-\mathrm{t}-\mathrm{Bu}]^{+}, 240,152,135,123,104,91,78$ (Found: $[\mathrm{M}]^{+}, 312.1539 . \mathrm{C}_{20} \mathrm{H}_{24} \mathrm{OS}$ requires $[\mathrm{M}]^{+}, 312.1542$ ).

\section{$\left(S_{p}, R_{\mathrm{S}}\right)-(+)-4-t e r t-B u t y l s u l f i n y l[2.2]$ paracyclophane} $\left[\left(S_{\mathrm{p}}, R_{\mathrm{S}}\right)-5\right]$

$\mathrm{mp}=122-124{ }^{\circ} \mathrm{C} ;[\alpha]_{\mathrm{D}}+151.4\left(\mathrm{c} 1, \mathrm{CHCl}_{3}\right)$ (assumed $80 \%$ ee see text); $v_{\max }$ (film) 2970, 2927, 2852, 1587, 1474, 1459, $1432,1410,1175,1039,904,847$ and $805 \mathrm{~cm}^{-1} ; \delta_{\mathrm{H}}(500 \mathrm{MHz}$, $\left.\mathrm{CDCl}_{3}\right) 6.93(1 \mathrm{H}, \mathrm{d}, J=10.5 \mathrm{~Hz}, \mathrm{H}-13), 6.58(1 \mathrm{H}, \mathrm{d}, J=10.0$ Hz, H-12), 6.54-6.47 (5H, m, H-5, H-7, H-8, H-15, H-16), 4.35 $(1 \mathrm{H}, \mathrm{t}, J=14.5 \mathrm{~Hz}, \mathrm{H}-2$ endo), $3.37(1 \mathrm{H}, \mathrm{ddd}, J=12.5,13.0$, $7.0 \mathrm{~Hz}, \mathrm{H}-1$ endo), 3.22-2.98 (5H, m, H-1 exo, $2 \times \mathrm{H}-9$ and $2 \times$ $\mathrm{H}-10), 2.82-2.77$ (1H, m, H-2 exo), 1.05 (9H, s, $t$-Bu); $\delta_{\mathrm{C}}(125$ $\mathrm{MHz}, \mathrm{CDCl}_{3}$ ) 142.2 (C), 140.7 (C), 139.3 (C), 139.0 (C), 137.7 $(\mathrm{CH}), 135.7(\mathrm{CH}), 134.2(\mathrm{CH}), 133.3(\mathrm{CH}), 133.0(\mathrm{CH}), 132.7$ $(\mathrm{CH}), 132.6(\mathrm{CH}), 132.5(\mathrm{CH}), 56.5(\mathrm{C}), 36.1\left(\mathrm{CH}_{2}\right), 35.2$ $\left(\mathrm{CH}_{2}\right), 35.0\left(\mathrm{CH}_{2}\right), 34.2\left(\mathrm{CH}_{2}\right), 23.1\left(\mathrm{CH}_{3}\right) ; \mathrm{m} / z(\mathrm{EI}+) 256[\mathrm{M}-$

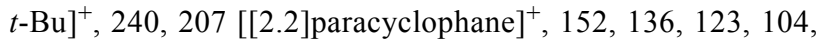
91, 78 (Found: $[\mathrm{M}]^{+}, 312.1545 . \mathrm{C}_{20} \mathrm{H}_{24} \mathrm{OS}$ requires $[\mathrm{M}]^{+}$, 312.1542).

\section{$\left(S_{p}\right)-(+)-4$-tert-Butylsulfanyl[2.2]paracyclo- phane $\left[\left(S_{p}\right)-6\right]$}

Triethylamine (11.92 $\mathrm{mL}, 85.58 \mathrm{mmol}, 10$ equiv) was added to a solution of trichlorosilane (17.9 mL, $128.36 \mathrm{mmol}, 15$ equiv) and $\left(S_{\mathrm{p}}, R_{\mathrm{S}}\right)-(+)$-4-tert-butylsulfinyl[2.2]paracyclophane $(2.67 \mathrm{~g}$, $8.56 \mathrm{mmol}, 1.0$ equiv) in toluene $(41 \mathrm{~mL})$. The reaction was heated to reflux for 18 hours. After cooling to $0{ }^{\circ} \mathrm{C}$ a solution of aqueous $\mathrm{NaOH}(3.0 \mathrm{M} ; 200 \mathrm{~mL})$ was added carefully. The 
aqueous phase was extracted with $\mathrm{Et}_{2} \mathrm{O}(3 \times 100 \mathrm{~mL})$ and the combined organic phases dried $\left(\mathrm{MgSO}_{4}\right)$. After removal of the solvent, the residue was purified by chromatography $\left(5 \% \mathrm{Et}_{2} \mathrm{O} /\right.$ hexane) followed by trituration of the yellow semi-solid with petrol gave $\left(S_{\mathrm{p}}\right)-6$ as a white solid $(1.0 \mathrm{~g}, 41.0 \%) ; \mathrm{mp}=53{ }^{\circ} \mathrm{C}$; $[\alpha]_{\mathrm{D}}+89.5\left(c 1, \mathrm{CHCl}_{3}\right)$ (assumed $80 \%$ ee see text); $v_{\max }($ film) 2957, 2926, 2894, 1471, 1455, 1433, 1411, 1389, 1362 and $1165 \mathrm{~cm}^{-1} ; \delta_{\mathrm{H}}\left(500 \mathrm{MHz}, \mathrm{CDCl}_{3}\right) 6.68(1 \mathrm{H}, \mathrm{s}, \mathrm{H}-5), 6.68(1 \mathrm{H}$, d, $J=10.0 \mathrm{~Hz}, \mathrm{H}-13), 6.54(2 \mathrm{H}, \mathrm{dd}, J=8.0,3.5 \mathrm{~Hz}, \mathrm{H}-7, \mathrm{H}-8)$, $6.50(2 \mathrm{H}, \mathrm{s}, \mathrm{H}-15, \mathrm{H}-16), 6.45(1 \mathrm{H}, \mathrm{d}, J=8.0 \mathrm{~Hz}, 12-\mathrm{H}), 3.84$ $(1 \mathrm{H}, \mathrm{t}, J=11.0 \mathrm{~Hz}, \mathrm{H}-2$ endo $), 3.20-3.14(1 \mathrm{H}, \mathrm{m}, \mathrm{H}-1$ endo $)$ 3.12-2.97 (5H, m, H-1 exo, $2 \times \mathrm{H}-9,2 \times \mathrm{H}-10), 2.83(1 \mathrm{H}$, ddd, $J=12.0,5.5,3.7 \mathrm{~Hz}, \mathrm{H}-2$ exo $), 1.17(9 \mathrm{H}, \mathrm{s}, t-\mathrm{Bu}) ; \delta_{\mathrm{C}}(125$ $\mathrm{MHz}, \mathrm{CDCl}_{3}$ ) 145.7 (C), 144.4 (C), 139.9 (C), 139.4 (C), 139.1 (C), $134.2(\mathrm{CH}), 133.5(\mathrm{CH}), 133.0(\mathrm{CH}), 132.9(\mathrm{CH}), 132.8$ $(\mathrm{CH}), 132.5(\mathrm{CH}), 131.0(\mathrm{CH}), 46.3(\mathrm{C}), 35.4\left(\mathrm{CH}_{2}\right), 35.4$ $\left(\mathrm{CH}_{2}\right), 34.8\left(\mathrm{CH}_{2}\right), 34.7\left(\mathrm{CH}_{2}\right), 30.9\left(\mathrm{CH}_{3}\right) ; \mathrm{m} / z(\mathrm{EI}+) 296$ $[\mathrm{M}]^{+}, 240[\mathrm{M}-t-\mathrm{Bu}]^{+}, 207[\mathrm{M}-\mathrm{S} t-\mathrm{Bu}]^{+}, 136,104,91,78$ (Found: $[\mathrm{M}]^{+}, 296.1591 . \mathrm{C}_{20} \mathrm{H}_{24} \mathrm{~S}$ requires $[\mathrm{M}]^{+}, 296.1593$ ).

\section{$\left(S_{\mathrm{p}}\right)-(+)$-Thioacetic acid S-[2.2]paracyclo- phan-4-yl ester $\left[\left(S_{\mathrm{p}}\right)-7\right]$}

Boron tribromide $(0.36 \mathrm{~mL}, 3.77 \mathrm{mmol}, 1.1$ equiv) was added to a solution of $\left(S_{\mathrm{p}}\right)-(+)-4$ - $t$-butylsulfanyl[2.2]paracyclophane $\left(S_{\mathrm{p}}\right)$-6 (1.0 g, $3.43 \mathrm{mmol}, 1.0$ equiv) and acetyl chloride (1.7 $\mathrm{mL}, 24.01 \mathrm{mmol}, 7.0$ equiv) in toluene $(34.3 \mathrm{~mL})$ at $\mathrm{rt}$. The reaction was stirred for 1 hour then poured into a solution of ice cold saturated aqueous $\mathrm{NH}_{4} \mathrm{Cl}(200 \mathrm{~mL})$. Aqueous phase was extracted with $\mathrm{Et}_{2} \mathrm{O}(3 \times 50 \mathrm{~mL})$. The combined organic phase was washed with aqueous $\mathrm{Na}_{2} \mathrm{~S}_{2} \mathrm{O}_{3}(10 \% \mathrm{w} / \mathrm{v})(3 \times 50 \mathrm{ml})$, dried $\left(\mathrm{MgSO}_{4}\right)$ and concentrated. Purification by chromatography $\left(10 \% \mathrm{Et}_{2} \mathrm{O} /\right.$ hexane) afforded $\left(S_{\mathrm{p}}\right)-7$ as a white solid $(0.53 \mathrm{~g}, 55 \%) ; \mathrm{mp}=134-136{ }^{\circ} \mathrm{C} ;[\alpha]_{\mathrm{D}}+84.3\left(\mathrm{c} 1, \mathrm{CHCl}_{3}\right)$ (assumed 80\% ee see text); $v_{\max }$ (film) 2920, 2850, 1694, 1498, $1476,1447,1432,1408,1113,954,906,850$ and $792 \mathrm{~cm}^{-1} ; \delta_{\mathrm{H}}$ $\left(500 \mathrm{MHz}, \mathrm{CDCl}_{3}\right) 6.68(1 \mathrm{H}, \mathrm{d}, J=8.0 \mathrm{~Hz}, \mathrm{H}-13), 6.60(2 \mathrm{H}, \mathrm{s}$, H-15, H-16), 6.52 (3H, m, H-5, H-7, H-8), 6.42 (1H, d, $J=7.5$ $\mathrm{Hz}, \mathrm{H}-12), 3.41-3.35$ (1H, m, H-2 endo), 3.13-2.97 (5H, m, $\mathrm{H}-1$ endo, $2 \times \mathrm{H}-9,2 \times \mathrm{H}-10), 2.99(1 \mathrm{H}, \mathrm{t}, J=10.2 \mathrm{~Hz}, \mathrm{H}-1$ exo), 2.92-2.86 (1H, m, H-2 exo), $2.36(3 \mathrm{H}, \mathrm{s}, \mathrm{Me}) ; \delta_{\mathrm{C}}(125$ $\left.\mathrm{MHz}, \mathrm{CDCl}_{3}\right) 190.7$ (CO), 143.1 (C), 140.4 (C), 139.4 (C), $139.1(\mathrm{C}), 135.1(\mathrm{CH}), 135.0(\mathrm{CH}), 134.5(\mathrm{CH}), 133.2(\mathrm{CH})$, $133.1(\mathrm{CH}), 132.3(\mathrm{CH}), 130.2(\mathrm{CH}), 129.2(\mathrm{CH}), 35.4\left(\mathrm{CH}_{2}\right)$, $34.9\left(\mathrm{CH}_{2}\right), 34.6\left(\mathrm{CH}_{2}\right), 34.4\left(\mathrm{CH}_{2}\right), 30.2\left(\mathrm{CH}_{3}\right) ; \mathrm{m} / z(\mathrm{EI}+) 282$ $[\mathrm{M}]^{+}, 240[\mathrm{M}+\mathrm{H}-\mathrm{Ac}]^{+}, 207[[2.2] \text { paracyclophane }]^{+}, 178,136$, 104, 91, 78, 43 (Found: $[\mathrm{M}]^{+}, 282.1075 . \mathrm{C}_{18} \mathrm{H}_{18} \mathrm{OS}$ requires $\left.[\mathrm{M}]^{+}, 282.1073\right)$.

\section{$\left(S_{\mathrm{p}}\right)-(+)-[2.2]$ Paracyclophane-4-thiol $\left[\left(S_{\mathrm{p}}\right)-2\right]$}

To a solution of $\left(S_{\mathrm{p}}\right)-7(0.51 \mathrm{~g}, 1.80 \mathrm{mmol}, 1.0$ equiv $)$ in methanol $(18 \mathrm{~mL})$ was added $\mathrm{K}_{2} \mathrm{CO}_{3}(3.71 \mathrm{~g}, 26.84 \mathrm{mmol}, 15$ equiv $)$ and the reaction stirred for 3 hours at $\mathrm{rt}$. The reaction was poured into saturated aqueous $\mathrm{NH}_{4} \mathrm{Cl}$ solution $(100 \mathrm{~mL})$ and extracted with $\mathrm{Et}_{2} \mathrm{O}(3 \times 100 \mathrm{~mL})$. The combined organic layers were dried $\left(\mathrm{MgSO}_{4}\right)$ and concentrated before purification by chromatography $\left(1 \% \mathrm{Et}_{2} \mathrm{O} /\right.$ hexane $)$ gave $\left(S_{\mathrm{p}}\right)-2$ as a white solid $(0.14 \mathrm{~g}, 32 \%) ; \mathrm{mp}=144-146{ }^{\circ} \mathrm{C} ;[\alpha]_{\mathrm{D}}+164.0\left(\mathrm{c} 1, \mathrm{CHCl}_{3}\right)$ (assumed 80\% ee see text); $v_{\max }$ (film) 3011, 2929, 2848, 2558, 1587, 1548, 1499, 1480, 1449, 1432, 1411, 1060, 938, 897, 849, 804 and $791 \mathrm{~cm}^{-1} ; \delta_{\mathrm{H}}\left(500 \mathrm{MHz}, \mathrm{CDCl}_{3}\right) 7.21(1 \mathrm{H}, \mathrm{d}, J=7.5$ $\mathrm{Hz}, \mathrm{H}-13), 6.57(1 \mathrm{H}, \mathrm{d}, J=7.5 \mathrm{~Hz}, \mathrm{H}-8), 6.47(1 \mathrm{H}, \mathrm{d}, J=7.5$ $\mathrm{Hz}, \mathrm{H}-7), 6.43(1 \mathrm{H}, \mathrm{d}, J=7.5 \mathrm{~Hz}, \mathrm{H}-12), 6.40(2 \mathrm{H}, \mathrm{t}, J=7.5$ $\mathrm{Hz}, \mathrm{H}-15, \mathrm{H}-16), 6.22$ (1H, s, H-5), $3.41(1 \mathrm{H}, \mathrm{t}, J=12.0 \mathrm{~Hz}$, $\mathrm{H}-2$ endo $), 3.26(1 \mathrm{H}, \mathrm{ddd}, \mathrm{J}=13.0,6.0,3.9 \mathrm{~Hz}, \mathrm{H}-1$ endo $), 3.13$ (1H, s, S-H), 3.11-3.01 (4H, m, $2 \times \mathrm{H}-9,2 \times \mathrm{H}-10), 2.90-2.87$ $(1 \mathrm{H}, \mathrm{t}, J=9.0 \mathrm{~Hz}, \mathrm{H}-1$ exo $), 2.83-2.77(1 \mathrm{H}, \mathrm{m}, \mathrm{H}-2$ exo $) ; \delta_{\mathrm{C}}$ (125 MHz, $\left.\mathrm{CDCl}_{3}\right) 140.4(\mathrm{C}), 139.3(\mathrm{C}), 139.1(\mathrm{C}), 138.5(\mathrm{C})$, $135.8(\mathrm{CH}), 134.8(\mathrm{CH}), 133.4(\mathrm{CH}), 132.8(\mathrm{CH}), 131.9(\mathrm{CH})$, $131.5(\mathrm{CH}), 130.5(\mathrm{CH}), 127.7(\mathrm{CH}), 35.4\left(\mathrm{CH}_{2}\right), 34.9\left(\mathrm{CH}_{2}\right)$, $34.6\left(\mathrm{CH}_{2}\right), 33.1\left(\mathrm{CH}_{2}\right) ; \mathrm{m} / z(\mathrm{EI}+) 240[\mathrm{M}]^{+}, 207$ [[2.2]paracyclophane $]^{+}, 136,104,91,78$ (Found: $[\mathrm{M}]^{+}, 240.0969 . \mathrm{C}_{16} \mathrm{H}_{16} \mathrm{~S}$ requires $\left.[\mathrm{M}]^{+}, 240.0967\right)$.

\section{$\left(R_{\mathrm{p}}, R_{\mathrm{S}}\right)-(-)-4-$ tert-Butylsulfinyl-5-amino[2.2]- paracyclophane $\left[\left(R_{\mathrm{p}}, R_{\mathrm{S}}\right)-\mathbf{8}\right]$}

To a solution of $\left(R_{\mathrm{p}}, R_{\mathrm{S}}\right)-(-)-4-t$-butylsulfinyl[2.2]paracyclophane $\left[\left(R_{\mathrm{p}}, R_{\mathrm{S}}\right)-5\right](5.80 \mathrm{~g}, 18.6 \mathrm{mmol}, 1.0$ equiv) in THF (350 $\mathrm{mL})$ at $0{ }^{\circ} \mathrm{C}$ was added $n$-BuLi $(2.5 \mathrm{M}$ in hexanes; $16.5 \mathrm{~mL}$, $41.25 \mathrm{mmol}, 2.2$ equiv) dropwise over $30 \mathrm{~min}$ to give an orange solution. After $1 \mathrm{~h}$ tosyl azide (9.20 g, $46.70 \mathrm{mmol}, 2.5$ equiv) was added and the reaction warmed to rt over $18 \mathrm{~h}$. $\mathrm{NaBH}_{4}$ (6.45 g, 171 mmol, 9 equiv) and tetra- $n$-butyl ammonium iodide ( $6.31 \mathrm{~g}, 17.1 \mathrm{mmol}, 0.9$ equiv) were added and the reaction stirred for a further $24 \mathrm{~h}$ at $\mathrm{rt}$ whereupon a further portion of $\mathrm{NaBH}_{4}$ (2.80 g, 74.0 mmol, 4.0 equiv) was added. After further stirring at $\mathrm{rt}$ for 5 days the reaction was poured into saturated aqueous $\mathrm{NH}_{4} \mathrm{Cl}(250 \mathrm{~mL})$ causing effervescence. The aqueous phase was extracted with $\mathrm{Et}_{2} \mathrm{O}(500 \mathrm{~mL}+200 \mathrm{~mL})$ and the combined organic extracts dried $\left(\mathrm{MgSO}_{4}\right)$ and the solvent removed. The residue was purified by chromatography (neutralized silica gel $40 \% \mathrm{Et}_{2} \mathrm{O} / n$-heptane) to yield $\mathbf{8}$ as a pale yellow solid powder, which was recrystalised from $\mathrm{CHCl}_{3} /$ heptane $(4.00 \mathrm{~g}, 66 \%) ; \mathrm{mp}=130-132{ }^{\circ} \mathrm{C} ;[\alpha]_{\mathrm{D}}-118.0\left(\mathrm{c} 1, \mathrm{CHCl}_{3}\right)$ (assumed 80\% ee see text); $v_{\max }$ (film) 3430, 3055, 2987, 1637, $1421,1265,896,739$ and $705 \mathrm{~cm}^{-1} ; \delta_{\mathrm{H}}\left(500 \mathrm{MHz}, \mathrm{CDCl}_{3}\right)$ $7.15(1 \mathrm{H}, \mathrm{d}, J=8.0 \mathrm{~Hz}, \mathrm{H}-13), 6.89(1 \mathrm{H}, \mathrm{d}, J=7.5 \mathrm{~Hz}, \mathrm{H}-16)$, $6.63(1 \mathrm{H}, \mathrm{d}, J=8.0 \mathrm{~Hz}, \mathrm{H}-15), 6.42(1 \mathrm{H}, \mathrm{d}, J=8.0 \mathrm{~Hz}, \mathrm{H}-7)$, $6.35(1 \mathrm{H}, \mathrm{d}, J=7.5 \mathrm{~Hz}, \mathrm{H}-12), 6.01(1 \mathrm{H}, \mathrm{d}, J=7.5 \mathrm{~Hz}, \mathrm{H}-8)$, $5.62\left(2 \mathrm{H}, \mathrm{s}\right.$, broad, $\left.\mathrm{NH}_{2}\right), 3.46(1 \mathrm{H}, \mathrm{t}, J=12.0 \mathrm{~Hz}, \mathrm{H}-2$ endo $)$, 3.23-3.18 (1H, m, H-1 endo), 3.12-3.01 (4H, m, H-1 exo, H-9 endo, $2 \times \mathrm{H}-10), 2.71-2.62(2 \mathrm{H}, \mathrm{m}, \mathrm{H}-2$ exo, $\mathrm{H}-9$ exo $), 1.19$ $(9 \mathrm{H}, \mathrm{s}, t-\mathrm{Bu}) ; \delta_{\mathrm{C}}\left(125 \mathrm{MHz}, \mathrm{CDCl}_{3}\right) 150.6(\mathrm{C}), 141.6(\mathrm{C}), 138.5$ 
(C), 138.1 (C), $133.1(\mathrm{C}), 132.1(\mathrm{C}), 131.9(\mathrm{CH}), 129.7$ (CH), $127.1(\mathrm{CH}), 126.5(\mathrm{CH}), 123.0(\mathrm{CH}), 114.7(\mathrm{CH}), 60.19(\mathrm{C})$, $34.1\left(\mathrm{CH}_{2}\right), 33.9\left(\mathrm{CH}_{2}\right), 32.8\left(\mathrm{CH}_{2}\right), 30.8\left(\mathrm{CH}_{2}\right), 23.6\left(\mathrm{CH}_{3}\right)$;

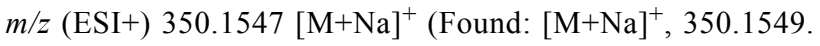
$\mathrm{C}_{20} \mathrm{H}_{25} \mathrm{OSNNa}$ requires $\left.[\mathrm{M}+\mathrm{Na}]^{+}, 350.1546\right)$.

\section{$\left(R_{\mathrm{p}}\right)$-(-)-4-tert-Butylsulfanyl-5-amino[2.2]- paracyclophane $\left[\left(R_{\mathrm{p}}\right)-9\right]$}

Triethylamine (1.47 mL, $10.53 \mathrm{mmol}, 10$ equiv) followed by trichlorosilane $(2.60 \mathrm{~mL}, 25.76 \mathrm{mmol}, 15.0$ equiv) were added carefully to a solution of $\left(R_{\mathrm{p}}, R_{\mathrm{S}}\right)-4-(-)-t$-butylsulfinyl-5amino[2.2]paracyclophane $\left[\left(R_{\mathrm{p}}, R_{\mathrm{S}}\right)-8\right](0.50 \mathrm{~g}, 1.61 \mathrm{mmol}, 1.0$ equiv) in toluene $\left(8 \mathrm{~mL}\right.$, ) at $0{ }^{\circ} \mathrm{C}$ and the reaction heated to reflux for $16 \mathrm{~h}$. The reaction was cooled to $0{ }^{\circ} \mathrm{C}$ and aqueous $\mathrm{NaOH}(3 \mathrm{M} ; 100 \mathrm{~mL})$ was added. The aqueous phase was extracted with $\mathrm{Et}_{2} \mathrm{O}(3 \times 50 \mathrm{~mL})$, dried $\left(\mathrm{MgSO}_{4}\right)$ and concentrated to give a pale yellow solid. Purification by chromatography (neutralized silica, 20\% $\mathrm{Et}_{2} \mathrm{O} /$ hexane) gave $\left(R_{\mathrm{p}}\right)-9$ as a pale yellow solid $(0.19 \mathrm{~g}, 40 \%) ; \mathrm{mp}=72-74{ }^{\circ} \mathrm{C} ;[\alpha]_{\mathrm{D}}-192.3(\mathrm{c}$ $1, \mathrm{CHCl}_{3}$ ) (assumed $80 \%$ ee see text); $v_{\max }$ (film) 3472, 3364, 2931, 2856, 1592, 1464, 1456, 1429, 1408, 1155, 876, 802, 741 and $717 \mathrm{~cm}^{-1} ; \delta_{\mathrm{H}}\left(500 \mathrm{MHz}, \mathrm{CDCl}_{3}\right) 7.0(1 \mathrm{H}, \mathrm{d}, J=9.5 \mathrm{~Hz}$, H-13), $6.60(1 \mathrm{H}, \mathrm{d}, J=10.0 \mathrm{~Hz}, \mathrm{H}-7), 6.47(1 \mathrm{H}, \mathrm{d}, J=10.0 \mathrm{~Hz}$, $\mathrm{H}-12), 6.41(1 \mathrm{H}, \mathrm{d}, J=9.5 \mathrm{~Hz}, \mathrm{H}-8), 6.33(1 \mathrm{H}, \mathrm{d}, J=9.5 \mathrm{~Hz}$, $\mathrm{H}-15), 6.23(1 \mathrm{H}, \mathrm{d}, J=9.5 \mathrm{~Hz}, \mathrm{H}-16), 4.47\left(2 \mathrm{H}, \mathrm{br} \mathrm{s}, \mathrm{NH}_{2}\right)$, $3.70(1 \mathrm{H}, \mathrm{ddd}, J=14.0,9.6,3.2 \mathrm{~Hz}, \mathrm{H}-2$ endo $), 3.11-2.96(5 \mathrm{H}$, $\mathrm{m}, \mathrm{H}-1$ endo, $2 \times \mathrm{H}-9,2 \times \mathrm{H}-10), 2.78-2.65(2 \mathrm{H}, \mathrm{m}, \mathrm{H}-1$ exo, $\mathrm{H}-2$ exo), 1.21 (9H, s, $t$-Bu); $\delta_{\mathrm{C}}\left(125 \mathrm{MHz}, \mathrm{CDCl}_{3}\right) 149.4$ (C), $147.3(\mathrm{C}), 139.1(\mathrm{C}), 138.3(\mathrm{C}), 135.7(\mathrm{CH}), 133.0(\mathrm{CH}), 132.2$ $(\mathrm{CH}), 129.7(\mathrm{CH}), 126.3(\mathrm{CH}), 124.3(\mathrm{C}), 123.0(\mathrm{CH}), 117.9$ (C), $48.1(\mathrm{C}), 35.3\left(\mathrm{CH}_{2}\right), 34.1\left(\mathrm{CH}_{2}\right), 32.8\left(\mathrm{CH}_{2}\right), 32.3\left(\mathrm{CH}_{2}\right)$, $30.9\left(\mathrm{CH}_{3}\right) ; m / z(\mathrm{EI}+) 311[\mathrm{M}]^{+}, 255[\mathrm{M}+\mathrm{H}-t-\mathrm{Bu}]^{+}, 207$ [[2.2]paracyclophane $]^{+}, 151,106$ (Found: $[\mathrm{M}+\mathrm{H}]^{+}, 312.1791$. $\mathrm{C}_{20} \mathrm{H}_{26} \mathrm{NS}$ requires $\left.[\mathrm{M}+\mathrm{H}]^{+}, 312.1780\right)$.

\section{$\left(R_{\mathrm{p}}\right)-(+)-[2.2](4,7)$ Benzo[d]thiazoloparacyclo- phane $\left[\left(R_{\mathrm{p}}\right)-10\right]$}

To a solution of 9 (141 $\mathrm{mg}, 0.45 \mathrm{mmol}, 1.0$ equiv) and paraformaldehyde (54.5 mg, $1.82 \mathrm{mmol}, 2.0$ equiv) in dioxane $(5.0$ $\mathrm{mL})$ and water $(1.0 \mathrm{~mL})$ was added aqueous $\mathrm{HCl}_{\text {conc. }}(9.0 \mathrm{M}$; $0.108 \mathrm{~mL}$ ). Pyridine (0.19 mL, $1.82 \mathrm{mmol}, 2.0$ equiv) was added and the reaction mixture heated to reflux for $48 \mathrm{~h}$. The reaction mixture was poured into aqueous $\mathrm{NaOH}(3.0 \mathrm{M} ; 20$ $\mathrm{mL})$ and the aqueous phase extracted with $\mathrm{Et}_{2} \mathrm{O}(3 \times 30 \mathrm{~mL})$ The combined organic phases were dried $\left(\mathrm{MgSO}_{4}\right)$ and the solvent removed; purification by chromatography (neutralized silica gel, $50 \% \mathrm{Et}_{2} \mathrm{O} /$ heptane) furnished $\left(R_{\mathrm{p}}\right)-\mathbf{1 0}$ as a pale yellow powder $(36.3 \mathrm{mg}, 28 \%) ; \mathrm{mp}=144-146{ }^{\circ} \mathrm{C} ;[\alpha]_{\mathrm{D}}+67.4$ (c 1, $\mathrm{CHCl}_{3}$ ) (assumed $80 \%$ ee see text); $v_{\max }$ (film) 3467 , 3005, 2978, 2934, 2873, 1448, 1384, 1351, 1217 and 1111 $\mathrm{cm}^{-1} ; \delta_{\mathrm{H}}\left(500 \mathrm{MHz}, \mathrm{CDCl}_{3}\right.$; paracyclophane numbering) 8.89
$(1 \mathrm{H}, \mathrm{s}$, thiazole $\mathrm{CH}), 6.81(1 \mathrm{H}, \mathrm{d}, J=7.5 \mathrm{~Hz}, \mathrm{H}-13), 6.68(1 \mathrm{H}$, $\mathrm{d}, J=7.5 \mathrm{~Hz}, \mathrm{H}-12), 6.52(1 \mathrm{H}, \mathrm{d}, J=8.0 \mathrm{~Hz}, \mathrm{H}-16), 6.46(1 \mathrm{H}$, $\mathrm{d}, J=7.5 \mathrm{~Hz}, \mathrm{H}-15), 6.18(1 \mathrm{H}, \mathrm{d}, J=7.5 \mathrm{~Hz}, \mathrm{H}-7), 5.85(1 \mathrm{H}, \mathrm{d}$, $J=7.5 \mathrm{~Hz}, \mathrm{H}-8), 3.99-3.93(1 \mathrm{H}, \mathrm{m}, \mathrm{H}-2$ endo $), 3.27-3.21(1 \mathrm{H}$, $\mathrm{m}, \mathrm{H}-1$ endo $), 3.17-3.11(1 \mathrm{H}, \mathrm{m}, \mathrm{H}-9$ endo $), 3.06-2.99(5 \mathrm{H}, \mathrm{m}$, $\mathrm{H}-1$ exo, $\mathrm{H}-2$ exo, $\mathrm{H}-9$ exo, $2 \times \mathrm{H}-10) ; \delta_{\mathrm{C}}\left(125 \mathrm{MHz}, \mathrm{CDCl}_{3}\right)$ $154.4(\mathrm{CH}), 151.8(\mathrm{C}), 139.2(\mathrm{C}), 137.2(\mathrm{C}), 134.8(\mathrm{C}), 134.4$ (C), $132.8(\mathrm{C}), 132.3(\mathrm{CH}), 131.7(\mathrm{CH}), 130.5(\mathrm{CH}), 126.2$ $(\mathrm{CH}), 124.9(\mathrm{CH}), 35.0\left(\mathrm{CH}_{2}\right), 34.5\left(\mathrm{CH}_{2}\right), 33.5\left(\mathrm{CH}_{2}\right), 32.4$ $\left(\mathrm{CH}_{2}\right) ; m / z(\mathrm{EI}+) 265[\mathrm{M}]^{+}, 161,104,78$ (Found: [M] ${ }^{+}$, 265.0921. $\mathrm{C}_{17} \mathrm{H}_{15} \mathrm{NS}$ requires $\left.[\mathrm{M}]^{+}, 265.0920\right)$.

\section{Acknowledgments}

The authors would like to thank Massey University and the University of Sussex for financial support.

\section{References}

1. Gleiter, R.; Hopf, H., Eds. Modern Cyclophane Chemistry; Wiley-VCH: Weinheim, Germany, 2004.

2. Vögtle, F. Cyclophane Chemistry; Wiley: Chichester, U.K., 1993.

3. Amthor, S.; Lambert, C. J. Phys. Chem. A 2006, 110, 3495-3504. doi:10.1021/jp055098o

4. Ball, P. J.; Shtoyko, T. R.; Krause Bauer, J. A.; Oldham, W. J.; Connick, W. B. Inorg. Chem. 2004, 43, 622-632. doi:10.1021/ic0348648

5. Bazan, G. C. J. Org. Chem. 2007, 72, 8615-8635. doi:10.1021/jo071176n

6. Morisaki, Y.; Chujo, Y. Angew. Chem., Int. Ed. 2006, 45, 6430-6437. doi:10.1002/anie.200600752

7. Valentini, L.; Mengoni, F.; Taticchi, A.; Marrocchi, A.; Kenny, J. M. J. Mater. Chem. 2006, 16, 1622-1625. doi:10.1039/b600333h

8. Valentini, L.; Mengoni, F.; Taticchi, A.; Marrocchi, A.; Landi, S.; Minuti, L.; Kenny, J. M. New J. Chem. 2006, 30, 939-943. doi:10.1039/b601535b

9. El-Shaieb, K. M.; Mourad, A. F. E.; Hopf, H. ARKIVOC 2006, No. ii193-200.

10. Gibson, S. E.; Knight, J. D. Org. Biomol. Chem. 2003, 1, 1256-1269. doi:10.1039/b300717k

11. Rozenberg, V.; Sergeeva, E.; Hopf, H. Cyclophanes as Templates in Stereoselective Synthesis. In Modern Cyclophane Chemistry; Gleiter, R.; Hopf, H., Eds.; Wiley-VCH: Weinheim, Germany, 2004; pp 435-462. doi:10.1002/3527603964.ch17

12. Ortner, B.; Hübner, H.; Gmeiner, $P$. Tetrahedron: Asymmetry 2001, 12, 3205-3208. doi:10.1016/S0957-4166(01)00558-4

13. Ortner, B.; Waibel, R.; Gmeiner, $P$. Angew. Chem., Int. Ed. 2001, 40, 1283-1285. doi:10.1002/1521-3773(20010401)40:7<1283::AID-ANIE1283>3.0.CO; 2-\#

14. Schlotter, K.; Boeckler, F.; Hübner, H.; Gmeiner, P. J. Med. Chem. 2006, 49, 3628-3635. doi:10.1021/jm060138d

15. Rowlands, G. J. Org. Biomol. Chem. 2008, 6, 1527-1534. doi:10.1039/b800698a

16. Lohier, J.-F.; Foucoin, F.; Jaffres, P.-A.; Garcia, J. I.; Sopková-de Oliveira Santos, J.; Perrio, S.; Metzner, P. Org. Lett. 2008, 10, 1271-1274. doi:10.1021/ol800161m 
17. Kane, V. V.; Gerdes, A.; Grahn, W.; Ernst, L.; Dix, I.; Jones, P. G.; Hopf, H. Tetrahedron Lett. 2001, 42, 373-376.

doi:10.1016/S0040-4039(00)01992-4

18. Kreis, M.; Bräse, S. Adv. Synth. Catal. 2005, 347, 313-319. doi:10.1002/adsc.200404299

19. Nakamura, S.; Ito, Y.; Wang, L.; Toru, T. J. Org. Chem. 2004, 69, 1581-1589. doi:10.1021/jo035558e

20. Vargas-Diaz, M. E.; Lagunas-Rivera, S.; Joseph-Nathan, P.; Tamariz, J.; Zepeda, L. G. Tetrahedron Lett. 2005, 46, 3297-3300. doi:10.1016/j.tetlet.2005.03.104

21. Gonda, J. Angew. Chem., Int. Ed. 2004, 43, 3516-3524. doi:10.1002/anie.200301718

22. Dondoni, A. Angew. Chem., Int. Ed. 2008, 47, 8995-8997. doi:10.1002/anie.200802516

23. Majumdar, K. C.; Debnath, P. Tetrahedron 2008, 64, 9799-9820. doi:10.1016/j.tet.2008.07.107

24. van Lindert, H. C. A.; van Doorn, J. A.; Bakker, B. H.; Cerfontain, H. Recl. Trav. Chim. Pays-Bas 1996, 115, 167-178.

25. van Lindert, H. C. A.; Koeberg-Telder, A.; Cerfontain, H. Recl. Trav. Chim. Pays-Bas 1992, 111, 379-388.

26. Braddock, D. C.; MacGilp, I. D.; Perry, B. G. Adv. Synth. Catal. 2004, 346, 1117-1130. doi:10.1002/adsc.200404065

27. Menichetti, S.; Faggi, C.; Lamanna, G.; Marrocchi, A.; Minuti, L.; Taticchi, A. Tetrahedron 2006, 62, 5626-5631. doi:10.1016/j.tet.2006.03.101

28. Pelter, A.; Mootoo, B.; Maxwell, A.; Reid, A. Tetrahedron Lett. 2001, 42, 8391-8394. doi:10.1016/S0040-4039(01)01808-1

29. Marchand, A.; Maxwell, A.; Mootoo, B.; Pelter, A.; Reid, A. Tetrahedron 2000, 56, 7331-7338. doi:10.1016/S0040-4020(00)00644-X

30. Hou, X.-L.; Wu, X.-W.; Dai, L.-X.; Cao, B.-X.; Sun, J. Chem. Commun. 2000, 1195-1196. doi:10.1039/b002679o

31. Hitchcock, P. B.; Rowlands, G. J.; Parmar, R. Chem. Commun. 2005, 4219-4221. doi:10.1039/b507394d

32. Hitchcock, P. B.; Hodgson, A. C. C.; Rowlands, G. J. Synlett 2006, 2625-2628. doi:10.1055/s-2006-950432

33. Hitchcock, P. B.; Rowlands, G. J.; Seacome, R. J. Org. Biomol. Chem. 2005, 3, 3873-3876. doi:10.1039/b509994c

34. Reich, H. J.; Yelm, K. E. J. Org. Chem. 1991, 56, 5672-5679. doi:10.1021/jo00019a039

35. Ernst, L.; Wittkowski, L. Eur. J. Org. Chem. 1999, 1999, 1653-1663. doi:10.1002/(SICI)1099-0690(199907)1999:7<1653::AID-EJOC1653>3 .0.CO;2-R

36. Reich, H. J.; Cram, D. J. J. Am. Chem. Soc. 1969, 91, 3534-3543. doi:10.1021/ja01041a018

37. Weix, D. J.; Ellman, J. A. Org. Lett. 2003, 5, 1317-1320. doi:10.1021/ol034254b

38. Seacome, R. J. The synthesis and methodology development of novel [2.2]paracyclophane-based compounds. D.Phil. Thesis, University of Sussex, U.K., 2008.

39. Parmar, R. New methodology for the synthesis of enantiopure [2.2]paracyclophane derivatives, D.Phil. Thesis, University of Sussex, U.K., 2006.

40. Mancheño, O. G.; Priego, J.; Cabrera, S.; Arrayás, R. G.; Llamas, T.; Carretero, J. C. J. Org. Chem. 2003, 68, 3679-3686. doi:10.1021/jo0340657

41. Stuhr-Hansen, N. Synth. Commun. 2003, 33, 641-646. doi:10.1081/SCC-120015820
42. Ulrich, H. Product Class 19: 1,2- and 1,3-Thiaphospholes and Benzoannulated Analogues. In Hetarenes and Related Ring Systems: Five-Membered Hetarenes with One Chalcogen and One Additional Heteroatom; Schaumann, E., Ed.; Science of Synthesis, Vol. 11; Georg Thieme Verlag: Stuttgart - New York, 2002; pp 835-912.

43. Fu, G. C. Acc. Chem. Res. 2006, 39, 853-860. doi:10.1021/ar068115g 44. Fu, G. C. Acc. Chem. Res. 2004, 37, 542-547. doi:10.1021/ar030051b 45. Bräse, S.; Dahmen, S.; Hoefener, S.; Lauterwasser, F.; Kreis, M.; Ziegert, R. E. Synlett 2004, 2647-2669. doi:10.1055/s-2004-836029

46. Fu, G. C. Planar-chiral Heterocycles as Enantioselective Organocatalysts. In Asymmetric Synthesis - The Essentials; Christmann, M.; Bräse, S., Eds.; Wiley-VCH: Weinheim, Germany, 2007; pp 195-199.

47. Bräse, S. Planar Chiral Ligands Based on [2.2]Paracyclophanes. In Asymmetric Synthesis - The Essentials; Christmann, M.; Bräse, S., Eds.; Wiley-VCH: Weinheim, Germany, 2007; pp 67-71.

48. Royer, R.; Lechartier, J. P.; Demerseman, P. Bull. Soc. Chim. Fr. 1973, 11, 3017-3018.

\section{License and Terms}

This is an Open Access article under the terms of the Creative Commons Attribution License (http://creativecommons.org/licenses/by/2.0), which permits unrestricted use, distribution, and reproduction in any medium, provided the original work is properly cited.

The license is subject to the Beilstein Journal of Organic Chemistry terms and conditions:

(http://www.beilstein-journals.org/bjoc)

The definitive version of this article is the electronic one which can be found at: doi:10.3762/bjoc.5.9 